\title{
Quantum dots in micropillar cavities for scalable photonic applications
}

Moczaa-Dusanowska, Magdalena; Huber, Tobias; Dusanowski, ukasz; Gerhardt, Stefan; Jurkat, Jonathan; Pfenning, Andreas; Trotta, Rinaldo; Rastelli, Armando; Gregersen, Niels; Schneider, Christian Total number of authors:

11

Published in:

Frontiers in Optics 2019

Publication date:

2019

Document Version

Publisher's PDF, also known as Version of record

Link back to DTU Orbit

Citation (APA):

Moczaa-Dusanowska, M., Huber, T., Dusanowski, ., Gerhardt, S., Jurkat, J., Pfenning, A., Trotta, R., Rastelli, A., Gregersen, N., Schneider, C., \& Hofling, S. (2019). Quantum dots in micropillar cavities for scalable photonic applications. In Frontiers in Optics 2019 [FM3D.2] Optical Society of America (OSA).

\section{General rights}

Copyright and moral rights for the publications made accessible in the public portal are retained by the authors and/or other copyright owners and it is a condition of accessing publications that users recognise and abide by the legal requirements associated with these rights.

- Users may download and print one copy of any publication from the public portal for the purpose of private study or research.

- You may not further distribute the material or use it for any profit-making activity or commercial gain

- You may freely distribute the URL identifying the publication in the public portal 


\title{
Quantum dots in micropillar cavities for scalable photonic applications
}

\author{
Magdalena Moczała-Dusanowska ${ }^{1}$, Tobias Huber $^{1, \dagger}$, Lukasz Dusanowski $^{1}$, Stefan

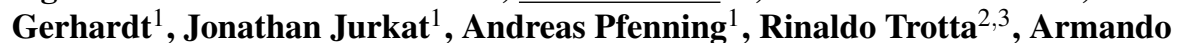 \\ Rastelli $^{3}$, Niels Gregersen ${ }^{4}$, Christian Schneider ${ }^{1}$, and Sven Höfling ${ }^{1,5}$ \\ ${ }^{1}$ Lehrstuhl für Technische Physik, Universität Würzburg, Am Hubland, 97074 Würzburg, Germany \\ ${ }^{2}$ Department of Physics, Sapienza University of Rome, Piazzale Aldo Moro, 5, 00185 Roma RM, Italy \\ ${ }^{3}$ Institute of Semiconductor and Solid State Physics, Johannes Kepler Universität Linz, Altenberger Straße 69, \\ 4040 Linz, Austria \\ ${ }^{4}$ Department of Photonics Engineering, Technical University of Denmark, Ørsteds Plads, 2800 Kgs. Lyngby, \\ Denmark ${ }^{5}$ SUPA, School of Physics and Astronomy, University of St Andrews, North Haugh, St Andrews KY16 \\ 9SS, UK \\ †tobias.huber@physik.uni-wuerzburg.de
}

\begin{abstract}
Scalable quantum photonic applications require wavelength reproducibility and high quality of the emitted photons. To address these issues, we investigate strain-tuning of self-assembled semiconductor quantum dots embedded into micropillar cavities. (C) 2019 The Author(s)
\end{abstract}

OCIS codes: $230.5590,300.6470,270.5580$

\section{Introduction}

Self-assembled semiconductor quantum dots are good sources of indistinguishable single photons [1] and entangled photon pairs [2]. Furthermore, the ground state spin of a trapped electron or hole can be used as a spin qubit [3]. Besides relatively short spin coherence times [4], one of the main drawbacks of quantum dots is the inhomogeneous distribution of the emission wavelength, due to the randomness of the growth process. For any kind of quantum gate with photons, the emitted photons have to be indistinguishable in all degrees of freedom, which includes their central emission wavelength. Experiments that include more than one quantum dot are subject to low device yield and are not scalable, because the aforementioned inhomogeneous wavelength distribution requires cherry picking of two (or more) quantum dots. This problem of random wavelengths has been recently solved by applying strain from various directions [5] on a device that did not include any kind of nanocavity. However, the highest quality emission in respect to indistinguishability of photons has been achieved when quantum dots were embedded into cavities [6].

Here, we show that quantum dots in micropillar cavities can also be tuned by strain, which enables their use in scalable applications. For example the scheme presented in Refs. [7,8], which is a first building block for a quantum repeater, could be upscaled to more than two, because the emitter is in a cavity, which makes it more efficient and even more importantly, it can be tuned.

\section{Results}

We fabricated an array of micropillars on a medium-density InAs quantum dot sample embedded into a $\lambda$ distributed Bragg reflector cavity, composed of 15 top and 25 bottom pairs of alternating AlAs and GaAs. The sample was thinned down to approximately $30 \mu \mathrm{m}$ and placed on a $\left[\mathrm{Pb}\left(\mathrm{Mg}_{1 / 3} \mathrm{Nb}_{2 / 3}\right) \mathrm{O}_{3}\right]_{0.72}-\left[\mathrm{PbTiO}_{3}\right]_{0.28}(\mathrm{PMN}-$ PT) piezoelectric substrate. The substrate was not micromachined for full control over the strain tensor as in Ref. [5], but instead a slab of piezoelectric material (as in Ref. [9]) was used. The micropillars were not fully etched through, but the etch stopped just below the cavity layer to ensure strain transfer into the quantum dots. By applying voltage to the piezoelectric substrate, the quantum dot emission can be tuned for about $0.5 \mathrm{~nm}(0.75$ $\mathrm{meV}$ ), see Fig. 1. The cavity resonance is not influenced by the applied voltage, as also visible in Fig. 1. The quality factors of the micropillar cavities is reduced compared to fully etched cavities. On some devices we could measure a Purcell factor of $\mathrm{F}_{\mathrm{P}}=4.4 \pm 0.7$.

The next step towards an useful implementation for quantum communication is to investigate the indistinguishability of distant sources, where either only one, or alternatively, also both sources are placed on piezoelectric substrates. As mentioned above, this will be a crucial step for any kind of gate and will also determine if the device can be used for some even more advanced experiments like the ones presented by Ref. [7,8]. 


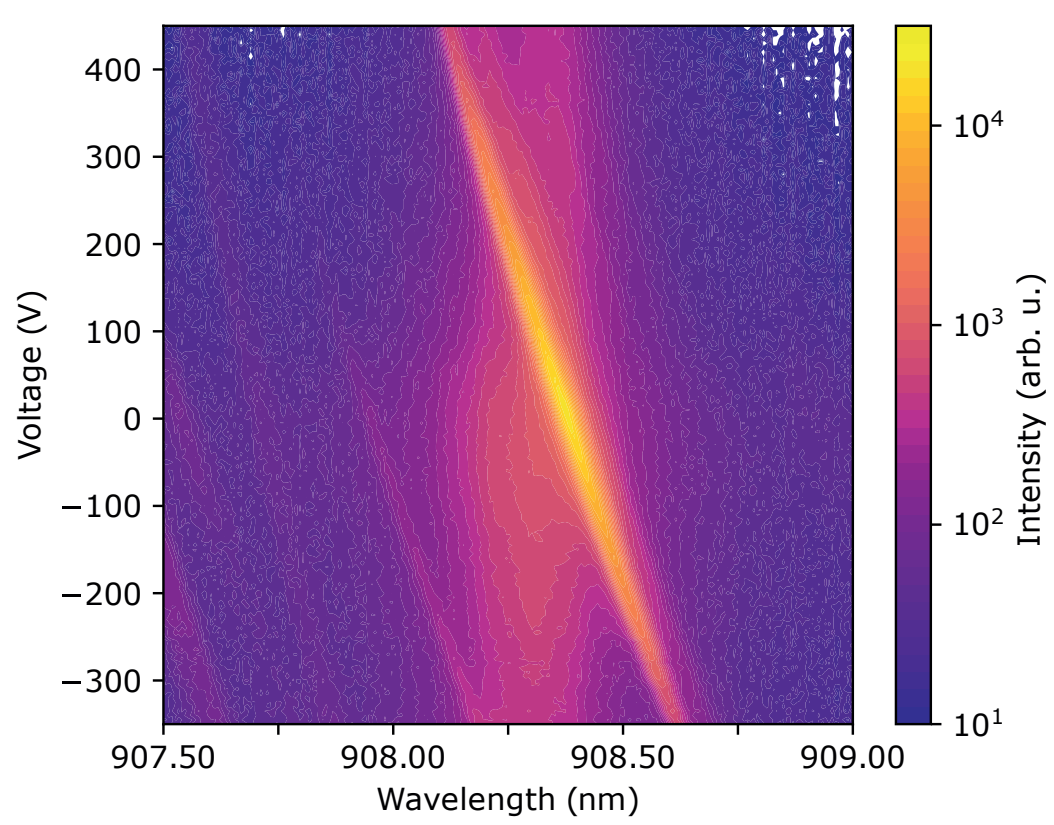

Fig. 1. Emission of a quantum dot embedded in a micropillar cavity on a piezoelectric substrate for different piezo voltages. The emission is plotted in logarithmic scale to make the faint cavity mode visible (broad feature at $908.3 \mathrm{~nm}$ ). The cavity mode is not influenced by the applied strain and stays constant, while the quantum dot emission can be tuned in and out of resonance.

\section{References}

1. Charles Santori, David Fattal, Jelena Vučković, Glenn S. Solomon, and Yoshihisa Yamamoto "Indistinguishable photons from a single-photon device," Nature 419, 594-597 (2002)

2. N. Akopian, N. H. Lindner, E. Poem, Y. Berlatzky, J. Avron, D. Gershoni, B. D. Gerardot, and P. M. Petroff "Entangled Photon Pairs from Semiconductor Quantum Dots," Phys. Rev. Lett. 96, 130501 (2006)

3. Richard J. Warburton "Single spins in self-assembled quantum dots," Nature Materials 12, 483-493 (2013)

4. L. Huthmacher, R. Stockill, E. Clarke, M. Hugues, C. Le Gall, and M. Atatüre, "Coherence of a dynamically decoupled quantum-dot hole spin, ’Phys. Rev. B 97, 241413(R) (2018)

5. R. Trotta, J. Martín-Sánchez, J. S. Wildmann, G. Piredda, M. Reindl, C. Schimpf, E. Zallo, S. Stroj, J. Edlinger, and A. Rastelli "Wavelength-tunable sources of entangled photons interfaced with atomic vapours," Nature Comm. 7, 10375 (2016)

6. Sebastian Unsleber, Yu-Ming He, Stefan Gerhardt, Sebastian Maier, Chao-Yang Lu, Jian-Wei Pan, Niels Gregersen, Martin Kamp, Christian Schneider, and Sven Höfling, "Highly indistinguishable on-demand resonance fluorescence photons from a deterministic quantum dot micropillar device with $74 \%$ extraction efficiency," Optics Express 8, 8539 (2016)

7. A. Delteil, Z. Sun, W. Gao, E. Togan, S. Faelt, and A. Imamoglu "Generation of heralded entanglement between distant hole spins," Nature Physics 12, 218 (2016)

8. R. Stockill, M. J. Stanley, L. Huthmacher, E. Clarke, M. Hugues, A. J. Miller, C. Matthiesen, C. Le Gall, and M. Atatüre "Phase-Tuned Entangled State Generation between Distant Spin Qubits," Phys. Rev. Lett. 119, 010503 (2017)

9. R. Trotta, P. Atkinson, J. D. Plumhof, E. Zallo, R. O. Rezaev, S. Kumar, S. Baunack, J. R. Schröter, A. Rastelli and O. G. Schmidt "Nanomembrane quantum-light-emitting diodes integrated onto piezoelectric actuators" Adv. Mater. 24 2668-72 (2012) 